\title{
Curcumin inhibits cancer-associated fibroblast-driven prostate cancer invasion through MAOA/mTOR/HIF-1 $\alpha$ signaling
}

\author{
YUEFENG DU ${ }^{1}$, QINGZHI LONG ${ }^{1}$, LIN ZHANG ${ }^{1}$, YING SHI $^{2}$, XIOAGANG LIU ${ }^{3}$, XUDONG LI $^{1}$,

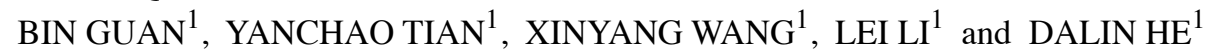 \\ ${ }^{1}$ Department of Urology, First Affiliated Hospital of Xi'an Jiaotong University, Xi'an, Shaanxi; ${ }^{2}$ Department of Urology, \\ Tongji Medical College Union Hospital, Huazhong University of Science and Technology, Wuhan, Hubei; \\ ${ }^{3}$ School of Life Science and Technology, The Key Laboratory of Biomedical Information Engineering \\ of Ministry of Education, Xi'an Jiaotong University, Xi'an, Shaanxi, P.R. China
}

Received August 2, 2015; Accepted September 25, 2015

DOI: $10.3892 /$ ijo.2015.3202

\begin{abstract}
Cancer-associated fibroblasts (CAFs) are key determinants in the malignant progression of cancer, supporting tumorigenesis and metastasis. CAFs also mediate epithelial to mesenchymal transition (EMT) in tumor cells and their achievement of stem cell traits. Curcumin has recently been found to possess anticancer activities via its effect on a variety of biological pathways involved in cancer progression. In this study, we found that CAFs could induce prostate cancer cell EMT and invasion through a monoamine oxidase A (MAOA)/ mammalian target of rapamycin (mTOR)/hypoxia-inducible factor-1 $\alpha$ (HIF-1 $\alpha)$ signaling pathway, which exploits reactive oxygen species (ROS) to drive a migratory and aggressive phenotype of prostate carcinoma cells. Moreover, CAFs was able to increase CXC chemokine receptor 4 (CXCR4) and interleukin-6 (IL-6) receptor expression in prostate cancer cells. However, curcumin abrogated CAF-induced invasion and EMT, and inhibited ROS production and CXCR4 and IL-6 receptor expression in prostate cancer cells through inhibiting MAOA/mTOR/HIF-1 $\alpha$ signaling, thereby supporting the therapeutic effect of curcumin in prostate cancer.
\end{abstract}

\section{Introduction}

Prostate cancer (PCa) is the second leading cause of male cancer death in the Western world (1). PCa patients can be clinically categorized into different risk groups primarily based on histological grade (Gleason score), clinical TNM stage, and levels of serum prostate specific antigen (2).

Correspondence to: Dr Dalin He, Department of Urology, First Affiliated Hospital of Xi'an Jiaotong University, 277 Yanta West Road, Xi'an, Shaanxi 710061, P.R. China

E-mail: dlhe2010@163.com

Key words: curcumin, cancer-associated fibroblasts, monoamine oxidase $\mathrm{A} / \mathrm{mammalian}$ target of rapamycin/hypoxia-inducible factor- $1 \alpha$ signaling, prostate cancer, invasion
Aggressive, poorly differentiated high-grade $\mathrm{PCa}$ is incurable and potentially lethal, underscoring the need for a greater understanding of the molecular basis of PCa progression and improved approaches to eliminating the development of the lethal phenotype of PCa.

Although tumorigenesis is largely acknowledged as a cell-autonomous process involving genetically transformed cancer cells, the importance of stromal cell types populating the tumor microenvironment is now well established $(3,4)$. Reactive stroma is composed of several heterotypic cells, including cancer associated fibroblasts (CAFs), macrophages, and endothelial cells. CAFs are phenotypically and functionally distinguishable from their normal counterparts based on their augmented proliferation and differential expression of extracellular matrix (ECM) proteins and soluble factors (5). Unlike resting fibroblasts, CAFs acquire an activated phenotype and can be recognized by their expression of fibroblast-specific protein 1 , desmin, vimentin, and $\alpha$-smooth muscle actin ( $\alpha$-SMA). To date, fibroblasts in tumor tissues are recognized as a diverse population of myofibroblastic cells intermixed with other fibroblasts that do not express $\alpha$-SMA, although they exert tumor promoting activity (6-9).

CAFs communicate among themselves as well as with cancer cells and inflammatory and immune cells directly through cell contact and indirectly through paracrine/exocrine signaling, proteases, and modulation of ECM. This complex communication network is pivotal in providing the appropriate microenvironment to support tumorigenesis, angiogenesis, and metastasis. Normal fibroblasts play a helpful role in maintaining epithelial homeostasis by suppressing proliferation of adjacent epithelia (10-12). After epithelial neoplastic transformation, CAFs undergo profound changes, promote tumor growth, induce angiogenesis, and recruit bone marrow-derived endothelial progenitor cells (13-15). Interestingly, CAFs can even mediate resistance to antiangiogenic therapy (16). In addition, CAFs affect the motility of cancer cells through remodeling of the ECM (17) and inducing epithelial to mesenchymal transition (EMT) of adjacent carcinoma cells (7). EMT is an epigenetic transcriptional program by which epithelial cells gain mesenchymal features as reduced cell-cell contacts and increased motility, thereby escaping the primary tumor 
and allowing dissemination of metastases to distant locations $(4,18)$. A hallmark of EMT is the loss of E-cadherin expression, in correlation with the tumor grade and stage (19-21).

Recently, Wu et al (22) reported that monoamine oxidase A (MAOA), a mitochondrial enzyme, mediates prostate tumorigenesis and cancer metastasis. MAOA functions to induce EMT and stabilize the transcription factor hypoxia-inducible factor $1 \alpha(\mathrm{HIF}-1 \alpha)$, which mediates hypoxia through an elevation of reactive oxygen species (ROS), thus enhancing the growth, invasiveness, and metastasis of PCa cells (22). In addition, the mammalian target of rapamycin (mTOR)-HIF-1 $\alpha$ pathway mediates aerobic glycolysis as a metabolic basis for trained immunity (23). Whether MAOA and the mTORHIF-1 $\alpha$ pathway play a role in the reciprocal activation of $\mathrm{PCa}$ cells and CAFs warrants further investigation.

Curcumin (diferuloylmethane) is a polyphenol derived from the Curcuma longa plant, commonly known as turmeric. Curcumin has been used extensively in Ayurvedic medicine for centuries $(24,25)$, as it is non-toxic and has a variety of therapeutic properties including anti-oxidant, analgesic, antiinflammatory, and anti-septic activities (26). More recently, curcumin has been found to possess anticancer activities via its effect on a variety of biological pathways involved in mutagenesis, oncogene expression, cell cycle regulation, apoptosis, tumorigenesis, and metastasis (26). Curcumin has exhibited an effect on targeting mTOR signaling to inhibit cancer progression (27-29). In addition, curcumin affects a variety of growth factor receptors and cell adhesion molecules involved in tumor growth, angiogenesis, and metastasis (26).

Here, we investigated the role of MAOA/mTOR/HIF-1 $\alpha$ signaling in CAF-induced EMT and invasiveness in PCa cells and examined the potential protective effect of curcumin on CAF-driven PCa progression. We found that MAOA/mTOR/ HIF-1 $\alpha$ signaling mediated CAF-induced EMT, invasion, ROS production, and CXCR4 and IL-6 receptor expression in $\mathrm{PCa}$ cells and curcumin suppressed CAF-induced PCa cell invasion through MAOA/mTOR/HIF-1 $\alpha$ signaling.

\section{Materials and methods}

Materials. The antibodies used in this study included polyclonal rabbit anti-human MAOA (Santa Cruz Biotechnology, Santa Cruz, CA, USA), monoclonal rabbit anti-human anti-mTOR (Cell Signaling Technology, Boston, MA, USA), polyclonal rabbit anti-human HIF-1 $\alpha$ (Bioworld, St. Louis Park, MN, USA), monoclonal mouse anti-human E-cadherin (Cell Signaling Technology), monoclonal mouse anti-human vimentin (Cell Signaling Technology), monoclonal mouse anti-human $\alpha$-SMA (Sigma-Aldrich, St. Louis, MO, USA), monoclonal mouse anti-human cytokeratin (Sigma-Aldrich), and monoclonal mouse anti-human $\beta$-actin (Santa Cruz Biotechnology). 2',7'-Dihydrochlorofluorescein diacetate ( $\mathrm{H}_{2}$ DCF-DA) was purchased from Molecular Probes (Carlsbad, CA, USA). Curcumin was purchased from Dolcas Biotech LLC (Landing, NJ, USA).

Cell cultures. PC3 cells were obtained from the Cell Bank of the Chinese Academy of Sciences (Shanghai, China). Human prostate CAFs were isolated from surgically explanted cancer regions of patients with $\mathrm{PCa}$ (Gleason 4+5) (30). Four different surgical explants were used for CAF isolation. The study protocol was approved by the relevant ethics committee of the First Affiliated Hospital of Medical College, Xi'an Jiaotong University, China, and the patients provided written informed consent. PC3 cells and fibroblasts were grown in Dulbecco's modified Eagle's medium containing $10 \%$ fetal bovine serum, $10 \mathrm{U} / \mathrm{ml}$ penicillin, and $10 \mathrm{mg} / \mathrm{ml}$ streptomycin. CAFs were used for 15 passages without significant modification of their ability to elicit EMT. Fresh serum-free medium was added for an additional $24 \mathrm{~h}$ before collection of conditioned medium (CM) in order to obtain CM free from CAFs. PC3 cells were then incubated with the obtained CM for $72 \mathrm{~h}$ and used for the analyses.

Western blot analysis. PC3 cells $\left(1 \times 10^{6}\right)$ grown under our experimental conditions were lysed for $20 \mathrm{~min}$ on ice in $300 \mu 1$ radioimmunoprecipitation assay (RIPA) lysis buffer [50 mM Tris- $\mathrm{HCl}$ (pH 7.5), $150 \mathrm{mM} \mathrm{NaCl}, 1 \%$ Triton X-100, $2 \mathrm{mM}$ EDTA, $1 \mathrm{mM}$ sodium orthovanadate, $1 \mathrm{mM}$ phenylmethanesulfonyl fluoride, $10 \mu \mathrm{g} / \mathrm{ml}$ aprotinin, and $10 \mu \mathrm{g} / \mathrm{ml}$ leupeptin]. Total proteins $(100 \mu \mathrm{g})$ were loaded onto sodium dodecyl sulfate (SDS)-polyacrylamide gel electrophoresis (PAGE) gels, separated, and transferred onto polyvinylidene fluoride (PVDF) membranes (Roche, Penzberg, Germany). The membranes were blocked with $5 \%$ non-fat dry milk in Tris-buffered saline with Tween-20 [TBST; $10 \mathrm{mM}$ Tris- $\mathrm{HCl}$ (pH 8.0), $150 \mathrm{mM} \mathrm{NaCl}, 0.05 \%$ Tween-20] and were subsequently incubated with primary antibodies overnight at $4{ }^{\circ} \mathrm{C}$. After five washes of $10 \mathrm{~min}$ each in TBST, the membranes were incubated with horseradish peroxidase (HRP)-conjugated secondary antibodies (1:3,000, Cell Signaling Technology) for $2 \mathrm{~h}$ and subsequently washed again. The peroxidase reaction was performed using an enhanced chemiluminescence detection system to visualize the immunoreactive bands.

Cell invasion assay. A chamber-based invasion assay (Millipore, Billerica, MA, USA) was performed to evaluate breast cancer cell invasion. Briefly, the upper surface of the membrane was coated with Matrigel (BD Biosciences, Franklin Lakes, NJ, USA). PC3 cells $\left(1 \times 10^{5}\right)$ were resuspended in the upper chamber in serum-free medium and allowed to migrate toward a serum gradient $(10 \%)$ in the lower chamber for $24 \mathrm{~h}$. The media was aspirated from inside the insert, and the non-invasive cells on the upper side were removed by a cotton swab. The membrane was fixed with $4 \%$ paraformaldehyde and stained with crystal violet. The number of migrated cells was counted on each membrane in 10 random fields and photographed at x100 magnification. The values reported here are the averages of triplicate experiments.

Quantitative real-time polymerase chain reaction assay (qRT-PCR). Total RNAs were extracted from PC3 cells using TRIzol reagent (Invitrogen, Carlsbad, CA, USA), and reverse transcription was performed using the PrimeScript RT Reagent kit (Takara, Dalian, China) according to the manufacturer's instructions. Real-time experiments were carried out using the iQ5 Multicolor Real-Time PCR Detection System (Bio-Rad, Hercules, CA, USA) and a SYBR Green PCR kit (Takara). The following PCR program was used: denaturation at $95^{\circ} \mathrm{C}$ for $30 \mathrm{sec}$, followed by 40 cycles consisting of denaturation at 
$95^{\circ} \mathrm{C}$ for $5 \mathrm{sec}$, annealing at $60^{\circ} \mathrm{C}$ for $30 \mathrm{sec}$, and extension at $72^{\circ} \mathrm{C}$ for $30 \mathrm{sec}$. A melting curve analysis was applied to assess the specificity of the amplified PCR products. The PCR primer sequences for HIF-1 $\alpha$ was 5'-AAGTCTAGGGATGCAGCA-3' (forward) and 5'-CAAGATCACCAGCATCATG-3' (reverse). The PCR primer sequences for MAOA was 5'-CTGATC GACTTGCTAAGCTAC-3' (forward) and 5'-ATGCACTGG ATGTAAAGCTTC-3' (reverse). The PCR primer sequences for mTOR was 5'-CTGGGACTCAAATGTGTGCAGTTC-3' (forward) and 5'-GAACAATAGGGTGAATGATCCGGG-3' (reverse). The PCR primer sequences for CXCR4 was 5'-TCTGTGACCGCTTCTACC-3' (forward) and 5'-AGGAT GAGGATGACTGTGG-3' (reverse). The PCR primer sequences for IL-6 receptor was 5'-CATTGCCATTGTTCTG AGGTTC-3' (forward) and 5'-AGTAGTCTGTATTGCTGAT GTC-3' (reverse). The PCR primer sequences for $\beta$-actin was 5'-TTGTTACAGGAAGTCCCTTGCC-3' (forward) and 5'-ATGCTATCACCTCCCCTGTGTG-3' (reverse). The amount of each target gene was quantified by the comparative $\mathrm{C}_{\mathrm{T}}$ method using $\beta$-actin as the normalization control (31).

Measurement of intracellular ROS. Intracellular production of $\mathrm{H}_{2} \mathrm{O}_{2}$ was assayed as previously described (32). Five minutes before the end of the incubation, the cells were treated with $5 \mu \mathrm{g} / \mathrm{ml}$ 2'-7'-dichlorofluorescein diacetate (DCF-DA). After washing with phosphate-buffered saline (PBS), the cells were lysed in $1 \mathrm{ml}$ RIPA buffer and analyzed immediately by fluorimetric analysis at $510 \mathrm{~nm}$. The data were normalized to total protein content.

Immunofluorescence microscopy. After the designated treatment, CAFs were fixed with $4 \%$ paraformaldehyde for $10 \mathrm{~min}$ at room temperature, permeabilized in $0.5 \%$ Triton $\mathrm{X}-100$ for $10 \mathrm{~min}$, and blocked in $1 \%$ bovine serum albumin (BSA) for $1 \mathrm{~h}$. Fixed cells were then incubated with mouse anti-human$\alpha$-SMA antibodies (1:100) or mouse anti-human-cytokeratin antibodies $(1: 100)$ at $4^{\circ} \mathrm{C}$ overnight. Cells were washed and incubated with goat anti-mouse dylight 594 (red) IgG antibody (Qenshare Biological Inc., Xi'an, China) at 1:200 dilution for $60 \mathrm{~min}$. Nuclei were stained with DAPI for $5 \mathrm{~min}$. The cells were visualized using a fluorescent microscope (Observer A1, Carl Zeiss Microscopy GmbH, Jena, Germany) using appropriate excitation and emission filters at $\times 400$ magnification.

RNA interference. shRNA against MAOA (Santa Cruz Biotechnology, sc-35847-SH), shRNA against mTOR (Santa Cruz Biotechnology, sc-35409-SH), shRNA against HIF-1 $\alpha$ (Santa Cruz Biotechnology, sc-35561-SH), and a negative control shRNA (Santa Cruz Biotechnology, sc-108060) were obtained from GenePharm (Shanghai, China). PC3 cells $\left(2 \times 10^{5}\right.$ per well) were seeded in 6-well plates and transfected with shRNA. Silencing with shRNA was performed with Lipofectamine (Invitrogen) following the manufacturer's instructions. Silenced cells were selected by puromycin treatment, and a pool of stably transfected cells was generated in order to avoid clonal selection.

Statistical analysis. The data are presented as the mean \pm standard deviations (SD) values obtained from at least three independent experiments. Statistical analysis of the data was performed by Student's t-tests using SPSS software (version 13.0; SPSS, Chicago, IL, USA). P-values $<0.05$ were considered statistically significant.

\section{Results}

CAFs activate MAOA/mTOR/HIF-1 $\alpha$ expression to elicit EMT in PC3 cells. To assess the role of MAOA/mTOR/HIF-1 $\alpha$ signaling elicited by CAFs in the EMT of cells, we used PC3 cells, a line isolated from a bone metastasis of human $\mathrm{PCa}$ and CAFs. CAFs were obtained from the surgically explanted prostate of patients with PCa (Gleason 4+5). Expression of E-cadherin and cytokeratin was assessed to exclude epithelial contamination of CAFs (Fig. 1A).

In order to study the molecular pathways driving the CAF-induced EMT response in PC3 cells, we analyzed the $\mathrm{MAOA} / \mathrm{mTOR} / \mathrm{HIF}-1 \alpha$ pathway in PC3 cells treated with CM from CAFs. PC3 cells exhibited CAF-induced EMT with activation of the MAOA/mTOR pathway. In addition, CAF exposure led to activation of HIF-1 $\alpha$ (Fig. 1B), a known transcription factor normally induced by hypoxia but already reported to be activated in normoxia by inflammation in an mTOR-dependent manner (23). Silencing of MAOA, mTOR, or HIF-1 $\alpha$ in PC3 cells by shRNA (Fig. 1C) after exposure to CAFs indicated that this signaling pathway proceeds from MAOA to mTOR and then transduction to HIF-1 $\alpha$ (Fig. 2A). MAOA, mTOR, or HIF-1 $\alpha$ knockdown in PC 3 cells after exposure to CAFs revealed that this MAOA/mTOR/HIF- $1 \alpha$ axis plays a mandatory role in orchestrating CAF-induced EMT in PC3 cells. Indeed, silencing of MAOA, mTOR, or HIF-1 $\alpha$ individually reduced the invasiveness and expression of EMT markers (E-cadherin and vimentin) among PC 3 cells (Fig. 2B-D).

MAOA/mTOR/HIF-1 $\alpha$ signaling regulates CAF-induced ROS production and CXCR4 and IL- 6 receptor expression in PC3 cells. Inflammation and oxidative stress have already been linked to cancer progression and claimed to be responsible for enhanced malignancy $(33,34)$. Oxidative stress, due to deregulated intracellular ROS production, has been correlated to PCa carcinogenesis, androgen resistance, anchorage-independence, and resistance to anoikis $(35,36)$. We observed that exposure to CAFs led to an increase in the ROS content of PC3 cells (Fig. 3A). ROS production appears to be mainly due to MAOA/mTOR/HIF- $1 \alpha$ signaling, as indicated by the ability of MAOA, mTOR, or HIF-1 $\alpha$ knockdown to block CAF-induced ROS generation and the invasive phenotype (Figs. 2C-D and 3A).

Moreover, culture in CM from CAFs resulted in a significant increase in CXCR4 and IL-6 receptor expression in PC3 cells (Fig. 3B and C). However, silencing of MAOA, mTOR, or HIF-1 $\alpha$ by shRNA in PC3 cells abrogated the elevated CXCR4 and IL-6 receptor expression induced by CAF-derived CM (Fig. 3B and C), which indicates an important role for $\mathrm{MAOA} / \mathrm{mTOR} / \mathrm{HIF}-1 \alpha$ signaling in PC3 cells with regard to the regulation of chemotactic and inflammation responses to CAF-derived CM.

Curcumin inhibits CAF-induced EMT in PC3 cells by suppressing MAOA/mTOR/HIF-1 $\alpha$ signaling. Curcumin has 

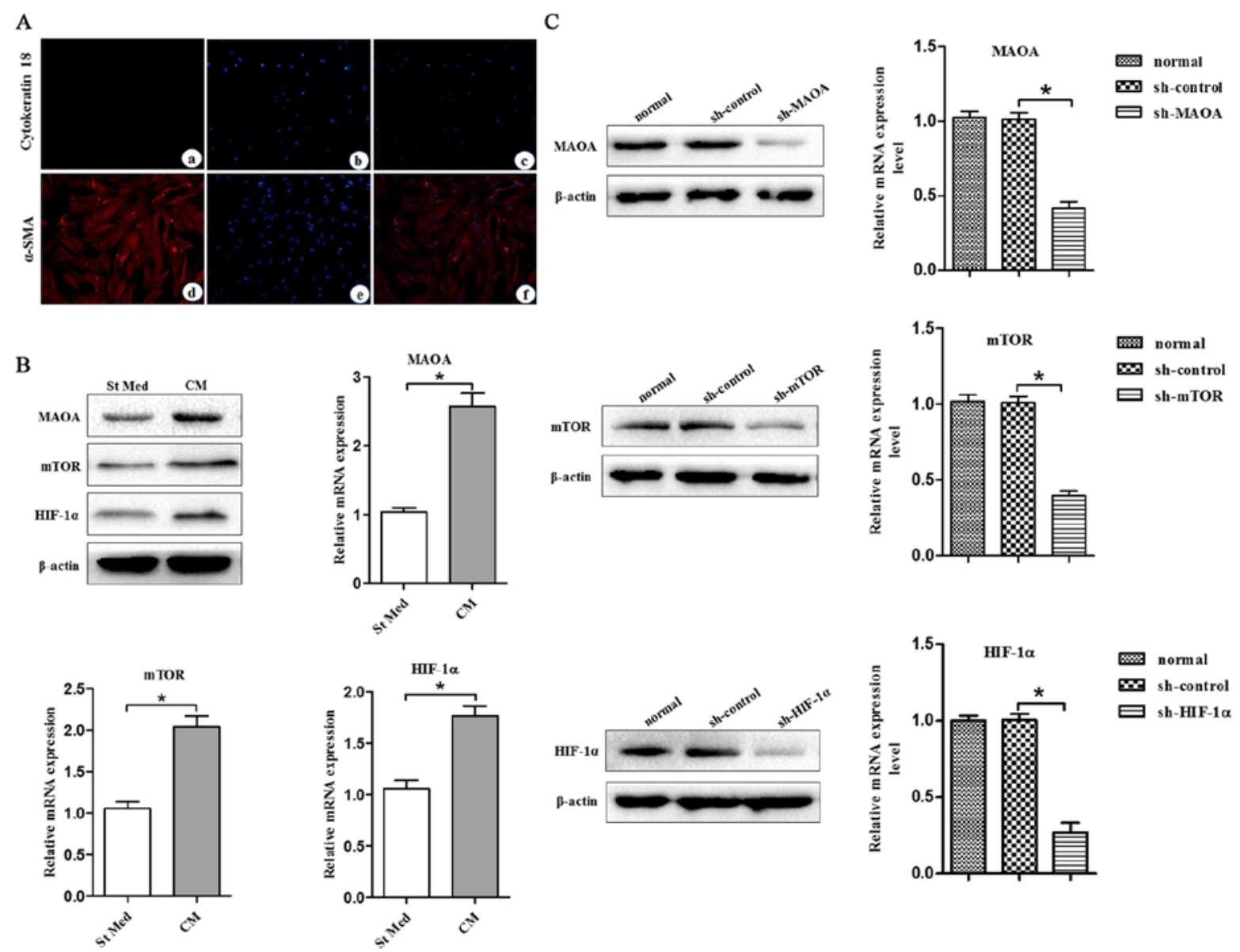

Figure 1. CAFs promote MAOA, mTOR, and HIF-1 $\alpha$ expression in PC3 cells. CM, conditioned media of CAFs, St Med, starved standard media of PC3 cells. CAFs were isolated from surgical explants from cancer regions of patients with PCa (Gleason 4+5). (A) (a-c) Cytokeratin 18 expression in CAFs was determined by immuofluorescence microscopy analysis. (d-f) $\alpha$-SMA expression in CAFs was determined by immuofluorescence microscopy analysis (B) PC3 cells were incubated with or without the indicated CM and the levels of MAOA, mTOR, HIF-1 $\alpha$, and $\beta$-actin were assessed by immunoblotting and qRT-PCR. (C) MAOA, mTOR, or HIF-1 $\alpha$ were transiently silenced with shRNA in PC3 cells. The interfering efficiency was determined by immunoblotting and qRT-PCR. "P<0.05.

A

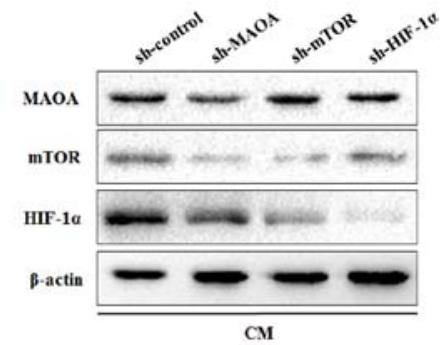

B

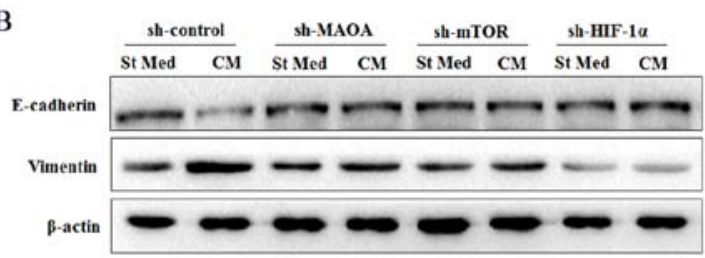

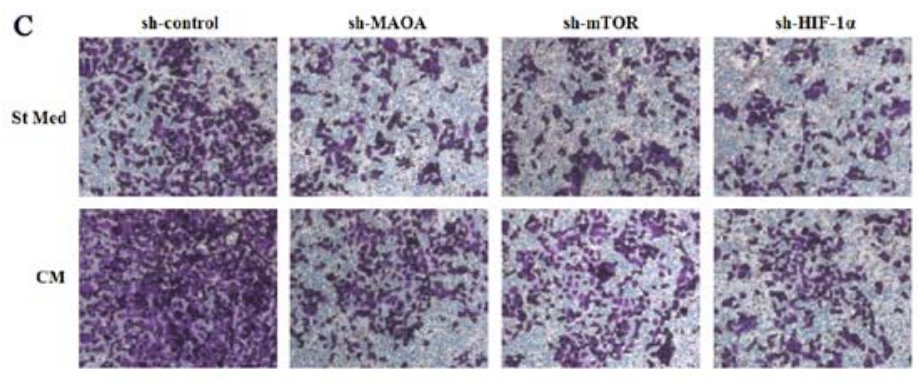

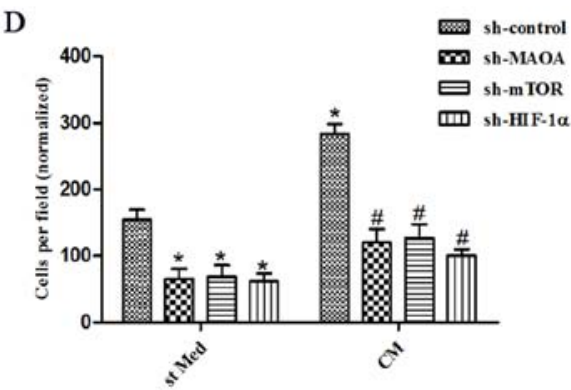

Figure 2. CAFs promote EMT by triggering a MAOA/mTOR/HIF-1 $\alpha$ signaling pathway. CM, conditioned media of CAFs, St Med, starved standard media of PC 3 cells. MAOA, mTOR, or HIF-1 $\alpha$ were transiently silenced with shRNA in PC3 cells. These cells were incubated with or without the indicated CM after silencing, and (A) the levels of MAOA, mTOR, HIF-1 $\alpha$, and $\beta$-actin were assessed by immunoblotting. (B) PC3 cells were treated as above, and the levels of E-cadherin, vimentin, and $\beta$-actin were assessed by immunoblotting. (C and D) PC3 cells were treated as above (B), and their invasion was evaluated. Invading cells were counted and a bar graph, representative of six randomly chosen fields, is shown. ${ }^{*} \mathrm{P}<0.05$ versus sh-control group treated with St Med group; ${ }^{*} \mathrm{P}<0.05$ versus sh-control group treated with CM. 
A

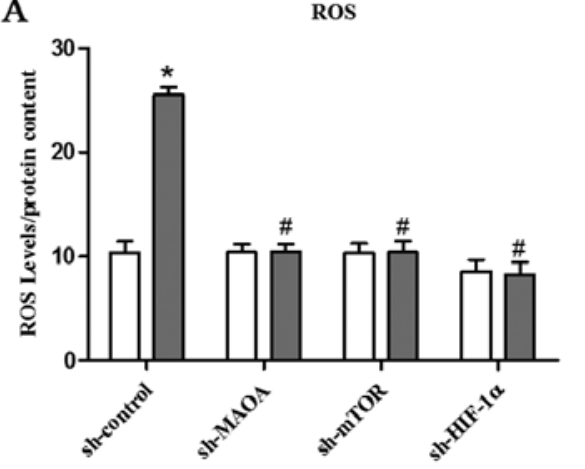

B

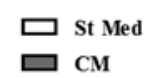

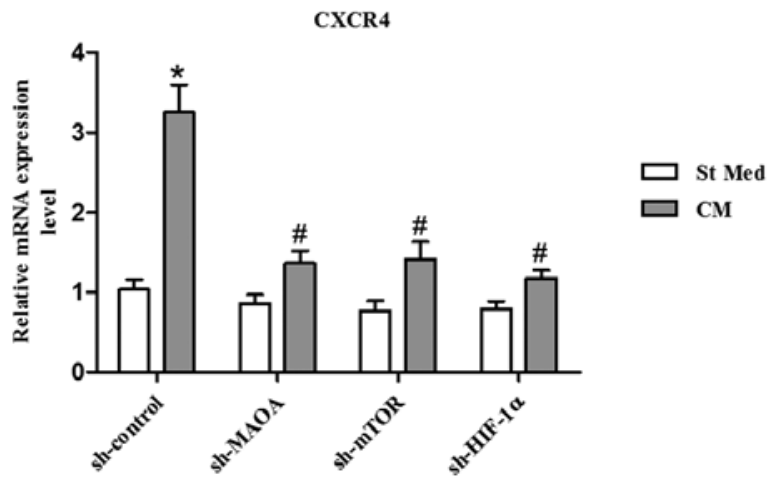

C

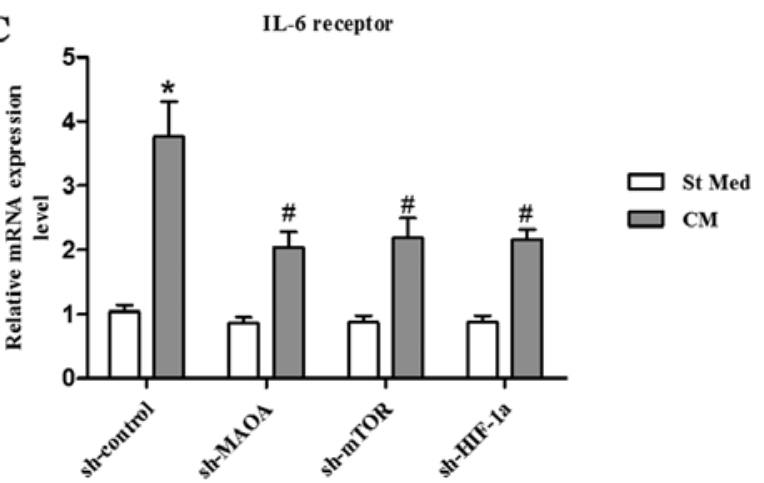

Figure 3. CAFs promote a MAOA/mTOR/HIF-1 $\alpha$-dependent oxidative response and CXCR4 and IL-6 receptor expression. CM, conditioned media of CAFs, St Med, starved standard media of PC 3 cells. MAOA, mTOR, or HIF-1 $\alpha$ were transiently silenced with shRNA in PC 3 cells, and these cells were incubated with or without the indicated CM, and (A) hydrogen peroxide production was evaluated with DCF-DA and normalized based on total protein content. CXCR4 (B) and IL-6 receptor (C) mRNA expression levels were evaluated by qRT-PCR. " $\mathrm{P}<0.05$ versus sh-control group treated with St Med group; ${ }^{*} \mathrm{P}<0.05$ versus sh-control group treated with $\mathrm{CM}$.

been found to possess anticancer activities based on its effect on a variety of biological pathways involved in mutagenesis, oncogene expression, cell cycle regulation, apoptosis, tumorigenesis, and metastasis; that is, the modulation of several important molecular targets including transcription factors (NF-кB, AP-1, $\beta$-catenin), enzymes (e.g., COX-2, MMPs), proinflammatory cytokines (e.g., TNF- $\alpha$, IL-1 $\beta$, and IL-6), and cell surface adhesion molecules (e.g., cadherins, integrins) (37-39). Here, we wanted to investigate whether curcumin has a protective effect in prostate cancer via modulation of MAOA/mTOR/HIF- $1 \alpha$ signaling.

We observed that $25 \mu \mathrm{M}$ curcumin [a concentration selected according to a previous study (40)] was able to abolish the EMT response to CAF-derived CM in PC3 cells as revealed by increased E-caderin levels and decreased vimentin levels (Fig. 4A). However, it could not inhibit the EMT response to CAF-derived $\mathrm{CM}$ in $\mathrm{PC} 3$ cells when MAOA, mTOR, or HIF-1 $\alpha$ was silenced by shRNA in PC3 cells (Fig. 4B). Similar results were obtained for the invasiveness of PC 3 cells. Curcumin abrogated the invasiveness of PC3 cells induced by CAF-derived CM (Fig. 4C). However, it was not able to suppress the invasiveness of $\mathrm{PC} 3$ cells induced by CAF-derived CM when MAOA, mTOR, or HIF-1 $\alpha$ was silenced by shRNA in PC3 cells (Fig. 4D).

Curcumin abrogates CAF-induced ROS production and CXCR4 and IL-6 receptor expression in PC3 cells through $M A O A / m T O R / H I F-1 \alpha$ signaling. Curcumin has been shown to have antioxidant activity and anti-inflammatory activity. Here, we aimed to evaluate the effect of curcumin on CAF-induced ROS production and CXCR4 and IL-6 receptor expression in PC3 cells. We found that curcumin abolished the CAF-derived CM-induced ROS production and CXCR4 and IL-6 receptor expression in PC3 cells (Fig. 5). However, it could not inhibit the CAFs in CM-induced ROS production and CXCR4 and IL-6 receptor expression in PC3 cells when MAOA, mTOR, or HIF-1 $\alpha$ was silenced by shRNA in PC3 cells (Fig. 5), which indicates that curcumin inhibits CAF-induced ROS production and CXCR4 and IL-6 receptor expression in PC 3 cells by impeding MAOA/mTOR/HIF-1 $\alpha$ signaling.

\section{Discussion}

The results presented in this report lead to three major conclusions: i) CAFs act on cancer cells by activating MAOA/mTOR/ HIF-1 $\alpha$ signaling, thus leading them to achieve a motile phenotype through EMT; ii) the proinflammatory signature (elevated CXCR4 and IL-6 receptor expression) activated in cancer cells is mainly driven by the pathway involving MAOA, mTOR, and HIF-1 $\alpha$; and iii) in response to CAF contact, cancer cells experience MAOA/mTOR/HIF-1 $\alpha$ signaling-mediated ROS accumulation.

MAOA has been associated with EMT activation. It has been shown that MAOA can stabilize HIF-1 $\alpha$, activate the vascular endothelial growth factor (VEGF)-A/neuropilin (NRP)1 system, and induce the expression of TWIST1, an 
A

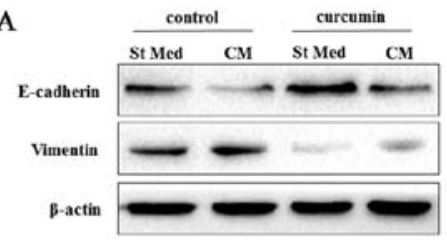

C

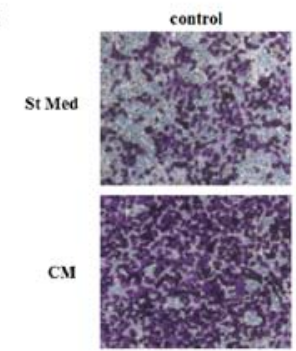

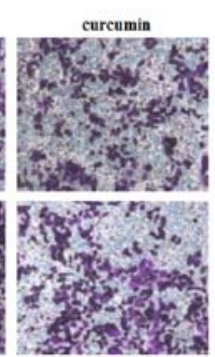
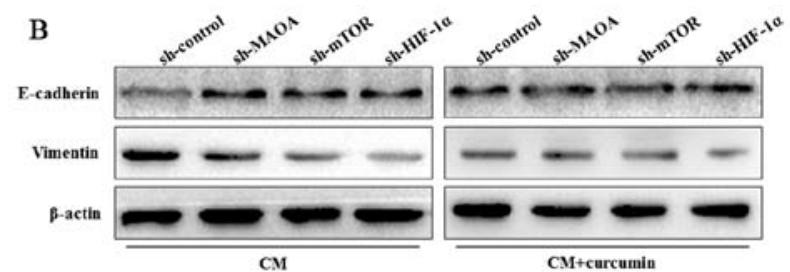

D
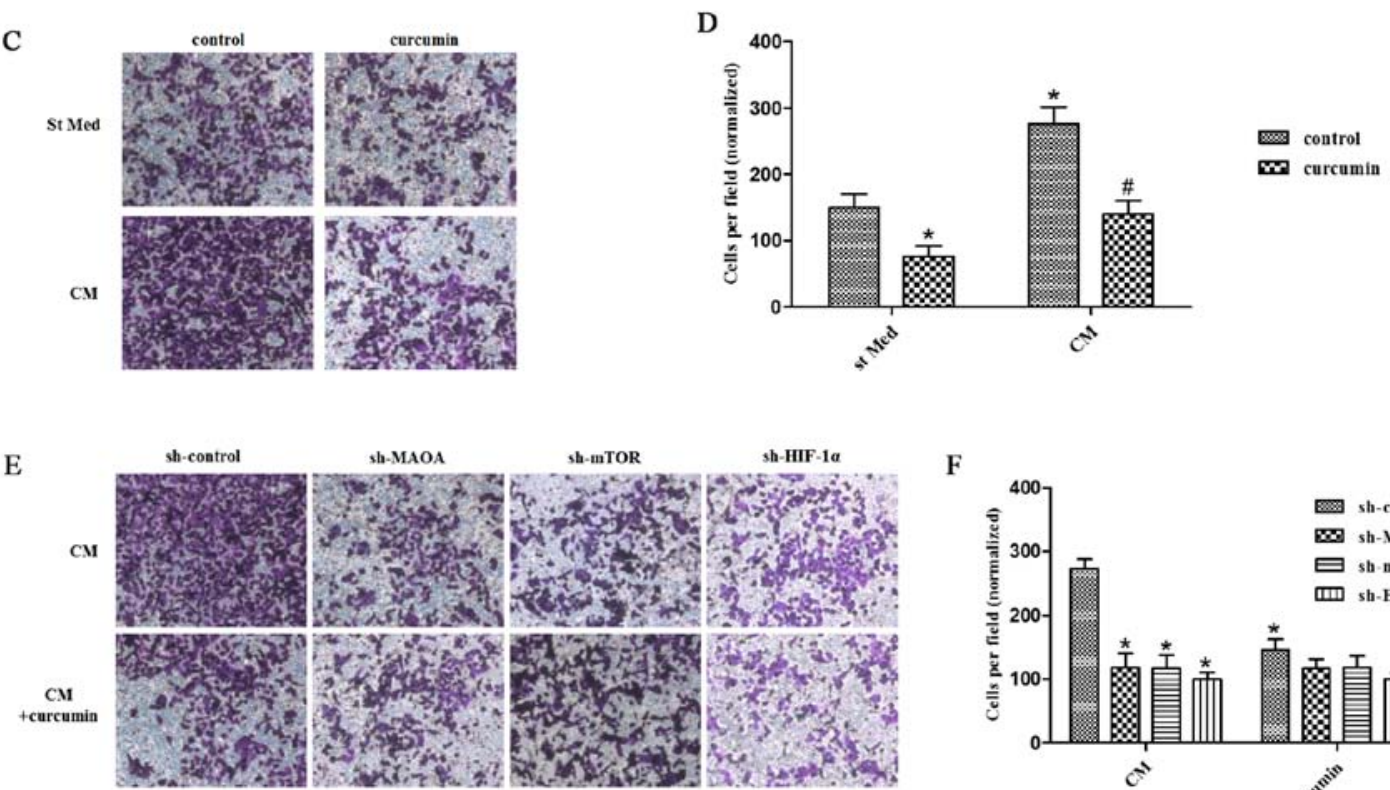

F

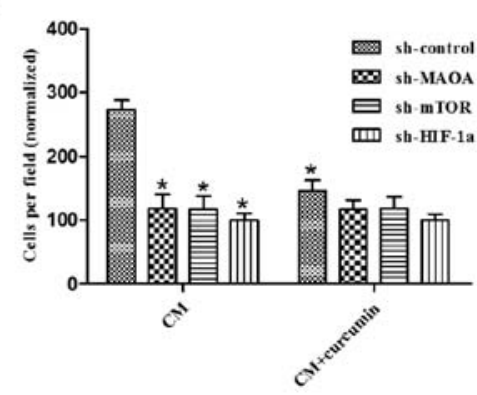

Figure 4. Curcumin suppresses CAF-induced EMT in PC3 cells via MAOA/mTOR/HIF-1 $\alpha$ signaling. CM, conditioned media of CAFs, St Med, starved standard media of PC3 cells. (A) PC3 cells were incubated with or without the indicated CM in the presence or absence of $25 \mu \mathrm{M}$ curcumin, and the levels of E-cadherin, vimentin, and $\beta$-actin were assessed by immunoblotting. (B) PC3 cells in which MAOA, mTOR, or HIF-1 $\alpha$ was transiently silenced with shRNA, and then were incubated with the indicated $\mathrm{CM}$ in the presence or absence of $25 \mu \mathrm{M}$ curcumin, and the levels of E-cadherin, vimentin, and $\beta$-actin were assessed by immunoblotting. (C and D) PC3 cells were treated as in (A), and their invasion was evaluated. Invading cells were counted and a bar graph, representative of six randomly chosen fields, is shown. ${ }^{*} \mathrm{P}<0.05$ versus control group treated with St Med; ${ }^{*} \mathrm{P}<0.05$ versus control group treated with $\mathrm{CM}$. (E and $\mathrm{F}$ ) PC 3 cells were treated as in (B), and their invasion was evaluated. Invading cells were counted and a bar graph, representative of six randomly chosen fields, is shown. ${ }^{*} \mathrm{P}<0.05$ versus sh-control group treated with $\mathrm{CM}$; $\mathrm{P}<0.05$ versus sh-control group treated with $\mathrm{CM}+$ curcumin.
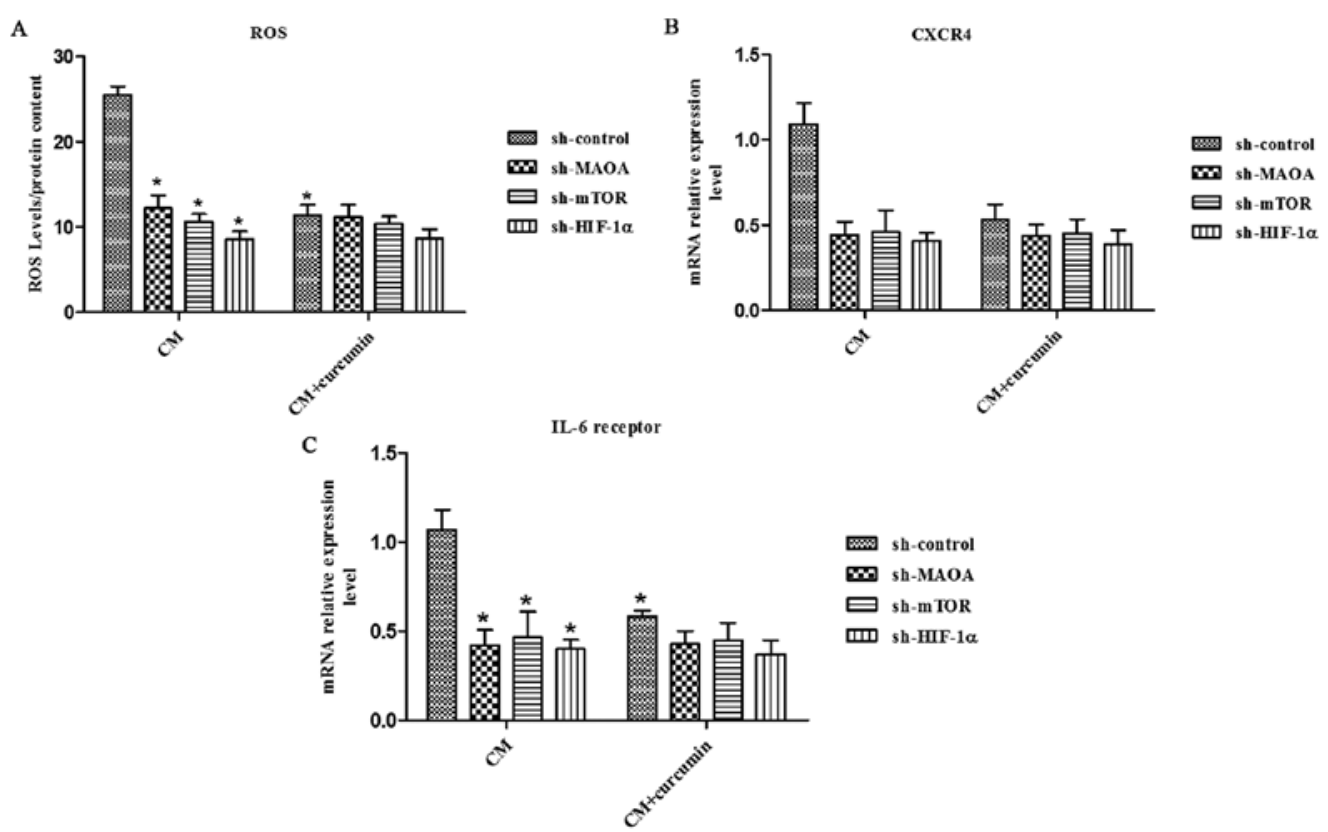

Figure 5. Curcumin abolishes CAF-induced ROS production and CXCR4 and IL-6 receptor expression in PC3 cells through the MAOA/mTOR/HIF-1 $\alpha$ signaling. CM, conditioned media of CAFs. PC3 cells in which MAOA, mTOR, or HIF-1 $\alpha$ was transiently silenced with shRNA were incubated with the indicated $\mathrm{CM}$ in the presence or absence of $25 \mu \mathrm{M}$ curcumin (A), hydrogen peroxide production was evaluated with DCF-DA and normalized based on total protein content. CXCR4 (B) and IL-6 receptor (C) mRNA expression levels were evaluated by qRT-PCR. "P<0.05 versus sh-control group treated with CM. 
EMT master transcription factor commonly associated with EMT promotion in PCa (22). Both increased MAOA expression and EMT were observed in the same specimens of high-grade $\mathrm{PCa}$, which indicate that MAOA can drive EMT in vivo (22). Pharmacological inhibition of MAOA in PCa cells kept basal prostatic epithelial cells from differentiating into matured glandular structures by reorganizing cell structures and decreasing the expression of basal cytokeratins (41). The CAF-induced EMT process in PCa appears to be mainly mediated by MAOA/mTOR/HIF-1 $\alpha$ signaling, as indicated by sensitivity of EMT suppression upon MAOA, mTOR, or HIF-1 $\alpha$ shRNA silencing.

Our results now include mTOR and HIF-1 $\alpha$ transcription factors as key players for CAF-mediated EMT activation. Recently, many studies have demonstrated that mTOR plays a crucial role in the regulation of cell motility, adhesion, and invasion (42-44). mTOR regulates EMT at least in part by downregulation of the RhoA and Rac1 signaling pathways (42) in PCa. In addition, HIF-1 $\alpha$ has been linked to the EMT process through the activation of both TWIST and Snail-1 transcription factors, leading to increased invasiveness $(45,46)$. We report herein the activation of HIF- $1 \alpha$ in response to CAF contact in normoxic conditions, which is mandatory for in vitro EMT activation. Consistent with our findings, activation of HIF-1 $\alpha$ also has been reported in normoxic conditions upon treatment with several growth factors and chemo-attractive agents $(47,48)$. Interestingly, EMT activation and chemotaxis surely share the activation of a migratory cell behavior, therefore allowing a correlation of non-hypoxic activation of HIF- $1 \alpha$ to the induction of a migratory behavior for PCa cells.

ROS can affect tumor progression in several ways. They cause oxidative damage to DNA and genomic instability or alter gene expression through modulation of transcription factors. In addition, repeated treatment with $\mathrm{H}_{2} \mathrm{O}_{2}$ caused a phenotypic conversion from mammary epithelial to fibroblastlike as in malignant transformation (49). ROS elicited by pancreatic stellate cells was shown to be able to induce EMT in pancreatic cancer cells (50). In PCa cells, ROS have also been correlated with EMT, through a MAOA-mediated delivery of mitochondrial ROS (22). Elevated ROS levels in MAOA-overexpressing cells contributed to increased HIF-1 $\alpha$ stabilization and activity, and the increased HIF-1 $\alpha$ expression has the potential to further induce mitochondrial activity including the formation of specific ROS during hypoxia $(51,52)$, potentially programming a 'vicious cycle' or feedforward loop among MAOA, ROS, and HIF-1 $\alpha$ to further drive PCa tumorigenesis. We demonstrate that MAOA, mTOR, or HIF-1 $\alpha$ silencing abrogated the ROS production increased by $\mathrm{CAF}$-derived $\mathrm{CM}$ exposure, which implicates a mandatory role for $\mathrm{MAOA} / \mathrm{mTOR} / \mathrm{HIF}-1 \alpha$ signaling in regulating CAF-induced ROS production.

CXCR4, which is involved in chemo-attraction of cancer and endothelial cells, and IL-6, which is involved in the organization of the pro-inflammatory response, have already been reported to be under transcriptional control of HIF-1 $(53,54)$. A recent study on pancreatic cancer showed that exogenous stromal cell-derived factor (SDF)-1 could induce CXCR4-positive pancreatic cancer invasion and EMT (55). Activated pancreatic cancer stellate cells secreted SDF-1 (the ligands that binds to the pro-inflammatory chemokine receptor CXCR4) and IL-6 to promote the pancreatic cancer EMT process. An active IL-6R/STAT3/miR-34a loop was necessary for EMT, invasion, and metastasis of colorectal cancer cell lines (56). Our data showed that exposure to CAFs increased CXCR4 and IL-6 receptor expression in PCa cells. These data indicate that PCa cells exposed to CAFs could be more easily attracted to different locations. Active factors in this chemo-attraction include CXCR4 and IL-6, confirming their pleiotropic role in $\mathrm{PCa}$ progression. Hence, the surrounding stroma might play a role in promoting metastasis of PCa cells from the primary lesions to the other organs, thereby facilitating satellite metastases.

Curcumin has been studied in multiple human carcinomas including melanoma, head and neck, breast, colon, pancreatic, prostate, and ovarian cancers (26). Curcumin can inhibit PCa growth and metastasis and increase the chemopreventive effects of other anticancer agents (57-60). Epidemiological studies attribute the low incidence of colon cancer in India to the chemopreventive and antioxidant properties of diets rich in curcumin (61). The mechanisms by which curcumin exerts its anticancer effects are comprehensive and diverse, targeting many levels of regulation in the processes of cellular growth and apoptosis. Here, we showed that curcumin inhibited PCa cell EMT and invasion induced by CAF-derived CM and abolished CAF-activated CXCR4 and IL-6 receptor expression in PCa cells. Moreover, curcumin abrogated ROS generation induced by exposure to CAFs in PCa cells. However, shRNAmediated downregulation of MAOA, mTOR, or HIF-1 $\alpha$ abolished the effects of curcumin on inhibiting PCa EMT and invasion. These data indicate that curcumin has a protective effect against the EMT process in the prostate tumor-stromal interaction, which is associated with its ability to ameliorate CAF-induced ROS production through the MAOA/mTOR/ HIF-1 $\alpha$ signaling pathway.

\section{Acknowledgements}

This study was supported by The National Science Foundation of China (nos. 81372736 and 81072107).

\section{References}

1. Siegel RL, Miller KD and Jemal A: Cancer statistics, 2015. CA Cancer J Clin 65: 5-29, 2015.

2. Partin AW, Kattan MW, Subong EN, Walsh PC, Wojno KJ, Oesterling JE, Scardino PT and Pearson JD: Combination of prostate-specific antigen, clinical stage, and Gleason score to predict pathological stage of localized prostate cancer. A multiinstitutional update. JAMA 277: 1445-1451, 1997.

3. Bissell MJ and Radisky D: Putting tumours in context. Nat Rev Cancer 1: 46-54, 2001.

4. Thiery JP: Epithelial-mesenchymal transitions in tumour progression. Nat Rev Cancer 2: 442-454, 2002.

5. Kalluri R and Zeisberg M: Fibroblasts in cancer. Nat Rev Cancer 6: 392-401, 2006.

6. Desmoulière A, Guyot $\mathrm{C}$ and Gabbiani G: The stroma reaction myofibroblast: A key player in the control of tumor cell behavior. Int J Dev Biol 48: 509-517, 2004.

7. Giannoni E, Bianchini F, Masieri L, Serni S, Torre E, Calorini L and Chiarugi P: Reciprocal activation of prostate cancer cells and cancer-associated fibroblasts stimulates epithelial-mesenchymal transition and cancer stemness. Cancer Res 70: 6945-6956, 2010.

8. Micke P and Ostman A: Tumour-stroma interaction: Cancerassociated fibroblasts as novel targets in anti-cancer therapy? Lung Cancer 45 (Suppl 2): S163-S175, 2004. 
9. Sugimoto H, Mundel TM, Kieran MW and Kalluri R: Identification of fibroblast heterogeneity in the tumor microenvironment. Cancer Biol Ther 5: 1640-1646, 2006.

10. Begley LA, Kasina S, MacDonald J and Macoska JA: The inflammatory microenvironment of the aging prostate facilitates cellular proliferation and hypertrophy. Cytokine 43: 194-199, 2008.

11. Bhowmick NA, Neilson EG and Moses HL: Stromal fibroblasts in cancer initiation and progression. Nature 432: 332-337, 2004.

12. Trimboli AJ, Cantemir-Stone CZ, Li F, Wallace JA, Merchant A, Creasap N, Thompson JC, Caserta E, Wang H, Chong JL, et al: Pten in stromal fibroblasts suppresses mammary epithelial tumours. Nature 461: 1084-1091, 2009.

13. Allinen M, Beroukhim R, Cai L, Brennan C, Lahti-Domenici J, Huang H, Porter D, Hu M, Chin L, Richardson A, et al: Molecular characterization of the tumor microenvironment in breast cancer. Cancer Cell 6: 17-32, 2004.

14. Olumi AF, Grossfeld GD, Hayward SW, Carroll PR, Tlsty TD and Cunha GR: Carcinoma-associated fibroblasts direct tumor progression of initiated human prostatic epithelium. Cancer Res 59: 5002-5011, 1999.

15. Orimo A, Gupta PB, Sgroi DC, Arenzana-Seisdedos F, Delaunay T, Naeem R, Carey VJ, Richardson AL and Weinberg RA: Stromal fibroblasts present in invasive human breast carcinomas promote tumor growth and angiogenesis through elevated SDF-1/CXCL12 secretion. Cell 121: 335-348, 2005.

16. Crawford Y and Ferrara N: Tumor and stromal pathways mediating refractoriness/resistance to anti-angiogenic therapies. Trends Pharmacol Sci 30: 624-630, 2009.

17. Pietras K, Pahler J, Bergers G and Hanahan D: Functions of paracrine PDGF signaling in the proangiogenic tumor stroma revealed by pharmacological targeting. PLoS Med 5: e19, 2008.

18. Friedl P: Prespecification and plasticity: Shifting mechanisms of cell migration. Curr Opin Cell Biol 16: 14-23, 2004.

19. Christofori G: New signals from the invasive front. Nature 441: 444-450, 2006

20. Cowin P, Rowlands TM and Hatsell SJ: Cadherins and catenins in breast cancer. Curr Opin Cell Biol 17: 499-508, 2005.

21. Francí C, Takkunen M, Dave N, Alameda F, Gómez S, Rodríguez R, Escrivà M, Montserrat-Sentís B, Baró T, Garrido M, et al: Expression of Snail protein in tumor-stroma interface. Oncogene 25: 5134-5144, 2006.

22. Wu JB, Shao C, Li X, Li Q, Hu P, Shi C, Li Y, Chen YT, Yin F, Liao CP, et al: Monoamine oxidase A mediates prostate tumorigenesis and cancer metastasis. J Clin Invest 124: 2891-2908, 2014.

23. Cheng SC, Quintin J, Cramer RA, Shepardson KM, Saeed S, Kumar V, Giamarellos-Bourboulis EJ, Martens JH, Rao NA, Aghajanirefah A, et al: mTOR- and HIF- $1 \alpha$-mediated aerobic glycolysis as metabolic basis for trained immunity. Science 345: $1250684,2014$.

24. Aggarwal BB, Sundaram C, Malani N and Ichikawa H: Curcumin: The Indian solid gold. Adv Exp Med Biol 595: 1-75, 2007.

25. Ammon HP and Wahl MA: Pharmacology of Curcuma longa. Planta Med 57: 1-7, 1991.

26. Wilken R, Veena MS, Wang MB and Srivatsan ES: Curcumin: A review of anti-cancer properties and therapeutic activity in head and neck squamous cell carcinoma. Mol Cancer 10: 12, 2011

27. Beevers CS, Zhou H and Huang S: Hitting the golden TORget: Curcumin's effects on mTOR signaling. Anticancer Agents Med Chem 13: 988-994, 2013.

28. Beevers CS, Chen L, Liu L, Luo Y, Webster NJ and Huang S: Curcumin disrupts the mammalian target of rapamycin-raptor complex. Cancer Res 69: 1000-1008, 2009

29. Yu S, Shen G, Khor TO, Kim JH and Kong AN: Curcumin inhibits Akt/mammalian target of rapamycin signaling through protein phosphatase-dependent mechanism. Mol Cancer Ther 7: 2609-2620, 2008.

30. Schnaitman C, Erwin VG and Greenawalt JW: The submitochondrial localization of monoamine oxidase. An enzymatic marker for the outer membrane of rat liver mitochondria. J Cell Biol 32: 719-735, 1967.

31. Schmittgen TD and Livak KJ: Analyzing real-time PCR data by the comparative C(T) method. Nat Protoc 3: 1101-1108, 2008.

32. Chiarugi P, Pani G, Giannoni E, Taddei L, Colavitti R, Raugei G, Symons M, Borrello S, Galeotti T and Ramponi G: Reactive oxygen species as essential mediators of cell adhesion: The oxidative inhibition of a FAK tyrosine phosphatase is required for cell adhesion. J Cell Biol 161: 933-944, 2003
33. Mantovani A: Role of inflammatory cells and mediators in tumor invasion and metastasis. Cancer Metastasis Rev 29: 241, 2010.

34. Pani G, Galeotti T and Chiarugi P: Metastasis: Cancer cell's escape from oxidative stress. Cancer Metastasis Rev 29: 351-378, 2010.

35. Giannoni E, Fiaschi T, Ramponi G and Chiarugi P: Redox regulation of anoikis resistance of metastatic prostate cancer cells: Key role for Src and EGFR-mediated pro-survival signals. Oncogene 28: 2074-2086, 2009.

36. Khandrika L, Kumar B, Koul S, Maroni P and Koul HK: Oxidative stress in prostate cancer. Cancer Lett 282: 125-136, 2009.

37. Kim HI, Huang H, Cheepala S, Huang S and Chung J: Curcumin inhibition of integrin (alpha6beta4)-dependent breast cancer cell motility and invasion. Cancer Prev Res (Phila) 1: 385-391, 2008.

38. Buhrmann C, Mobasheri A, Busch F, Aldinger C, Stahlmann R, Montaseri A and Shakibaei M: Curcumin modulates nuclear factor kappaB (NF-kappaB)-mediated inflammation in human tenocytes in vitro: Role of the phosphatidylinositol 3-kinase/Akt pathway. J Biol Chem 286: 28556-28566, 2011.

39. Shehzad A, Lee J and Lee YS: Curcumin in various cancers. Biofactors 39: 56-68, 2013.

40. Cheng TS, Chen WC, Lin YY, Tsai CH, Liao CI, Shyu HY, Ko CJ, Tzeng SF, Huang CY, Yang PC, et al: Curcumin-targeting pericellular serine protease matriptase role in suppression of prostate cancer cell invasion, tumor growth, and metastasis. Cancer Prey Res (Phila) 6: 495-505, 2013.

41. Zhao H, Nolley R, Chen Z, Reese SW and Peehl DM: Inhibition of monoamine oxidase A promotes secretory differentiation in basal prostatic epithelial cells. Differentiation 76: 820-830, 2008.

42. Chen X, Cheng H, Pan T, et al: mTOR regulate EMT through RhoA and Racl pathway in prostate cancer. Mol Carcinog 54: 1086-1095, 2014

43. Liu L, Li F, Cardelli JA, Martin KA, Blenis J and Huang S: Rapamycin inhibits cell motility by suppression of mTORmediated S6K1 and 4E-BP1 pathways. Oncogene 25: 7029-7040, 2006.

44. Gulhati P, Bowen KA, Liu J, Stevens PD, Rychahou PG, Chen M, Lee EY, Weiss HL, O'Connor KL, Gao T, et al: mTORC1 and mTORC2 regulate EMT, motility, and metastasis of colorectal cancer via RhoA and Rac1 signaling pathways. Cancer Res 71: 3246-3256, 2011.

45. Cannito S, Novo E, Compagnone A, Valfrè di Bonzo L, Busletta C, Zamara E, Paternostro C, Povero D, Bandino A, Bozzo F, et al: Redox mechanisms switch on hypoxia-dependent epithelial-mesenchymal transition in cancer cells. Carcinogenesis 29: 2267-2278, 2008

46. Yang MH and Wu KJ: TWIST activation by hypoxia inducible factor-1 (HIF-1): Implications in metastasis and development. Cell Cycle 7: 2090-2096, 2008

47. Calvani M, Rapisarda A, Uranchimeg B, Shoemaker RH and Melillo G: Hypoxic induction of an HIF-1alpha-dependent bFGF autocrine loop drives angiogenesis in human endothelial cells. Blood 107: 2705-2712, 2006.

48. Stasinopoulos I, O'Brien DR and Bhujwalla ZM: Inflammation, but not hypoxia, mediated HIF-1alpha activation depends on COX-2. Cancer Biol Ther 8: 31-35, 2009.

49. Mori K, Shibanuma M and Nose K: Invasive potential induced under long-term oxidative stress in mammary epithelial cells. Cancer Res 64: 7464-7472, 2004

50. Lei J, Huo X, Duan W, Xu Q, Li R, Ma J, Li X, Han L, Li W, Sun $\mathrm{H}$, et al: $\alpha$-Mangostin inhibits hypoxia-driven ROS-induced PSC activation and pancreatic cancer cell invasion. Cancer Lett 347: 129-138, 2014

51. Chandel NS, McClintock DS, Feliciano CE, Wood TM, Melendez JA, Rodriguez AM and Schumacker PT: Reactive oxygen species generated at mitochondrial complex III stabilize hypoxia-inducible factor-1alpha during hypoxia: A mechanism of $\mathrm{O}_{2}$ sensing. J Biol Chem 275: 25130-25138, 2000.

52. Chandel NS, Maltepe E, Goldwasser E, Mathieu CE, Simon MC and Schumacker PT: Mitochondrial reactive oxygen species trigger hypoxia-induced transcription. Proc Natl Acad Sci USA 95: 11715-11720, 1998

53. Semenza GL: Oxygen homeostasis. Wiley Interdiscip Rev Syst Biol Med 2: 336-361, 2010.

54. Youn SW, Lee SW, Lee J, Jeong HK, Suh JW, Yoon CH, Kang HJ, Kim HZ, Koh GY, Oh BH, et al: COMP-Ang1 stimulates HIF-1 $\alpha$-mediated SDF-1 overexpression and recovers ischemic injury through BM-derived progenitor cell recruitment. Blood 117: 4376-4386, 2011 
55. Li X, Ma Q, Xu Q, Liu H, Lei J, Duan W, Bhat K, Wang F, Wu E and Wang Z: SDF-1/CXCR4 signaling induces pancreatic cancer cell invasion and epithelial-mesenchymal transition in vitro through non-canonical activation of Hedgehog pathway. Cancer Lett 322: 169-176, 2012

56. Rokavec M, Öner MG, Li H, Jackstadt R, Jiang L, Lodygin D, Kaller M, Horst D, Ziegler PK, Schwitalla S, et al: IL-6R/STAT3/ miR-34a feedback loop promotes EMT-mediated colorectal cancer invasion and metastasis. J Clin Invest 124: 1853-1867, 2014.

57. Zhou DY, Ding N, Du ZY, Cui XX, Wang H, Wei XC, Conney AH, Zhang $\mathrm{K}$ and Zheng $\mathrm{X}$ : Curcumin analogues with high activity for inhibiting human prostate cancer cell growth and androgen receptor activation. Mol Med Rep 10: 1315-1322, 2014.
58. Dorai T, Diouri J, O'Shea O and Doty SB: Curcumin inhibits prostate cancer bone metastasis by up-regulating bone morphogenic protein-7 in vivo. J Cancer Ther 5: 369-386, 2014.

59. Eom DW, Lee JH, Kim YJ, et al: Synergistic effect of curcumin on epigallocatechin gallate-induced anticancer action in PC3 prostate cancer cells. BMB Rep 48: 461-466, 2015.

60. Wang P, Wang B, Chung S, Wu Y, Henning SM and Vadgama JV: Increased chemopreventive effect by combining arctigenin, green tea polyphenol and curcumin in prostate and breast cancer cells. RSC Advances 4: 35242-35250, 2014.

61. Mohandas KM and Desai DC: Epidemiology of digestive tract cancers in India. V. Large and small bowel. Indian J Gastroenterol 18: 118-121, 1999. 